\title{
Massive liver metastasis from colon adenocarcinoma causing cardiac tamponade
}

\author{
Aditya N Halthore, MD, ${ }^{a}$ Anna Levy, DO, ${ }^{\mathrm{b}}$ Tony Philip, MD, ${ }^{\mathrm{b}}$ and Sewit Teckie, MD \\ Departments of a Radiation Medicine and ${ }^{b}$ Medical Oncology, Hofstra Northwell School of Medicine, Lake Success, New York
}

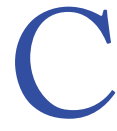

olorectal cancer is the third most commonly diagnosed cancer in the United States. ${ }^{1}$

About 5\% of Americans will be diagnosed with colorectal cancer in their lifetime, of which $20 \%$ will present with distant metastasis. ${ }^{2}$ The most common sites of metastasis are regional lymph nodes, liver, lung and peritoneum, and patients may present with signs or symptoms related to disease burden at any of these organs. In this case, we present a patient with metastatic colorectal cancer to liver who developed cardiac tamponade due to extrinsic compression from an enlarging liver lesion. We are not aware of this unusual complication being reported elsewhere in the literature and we discuss our approach to this challenging case.

\section{Case presentation and summary}

A 55-year-old man had presented to an outside hospital in August of 2014 with 6 months of hematochezia and a 40-lb weight loss. He was found to be severely anemic on admission (hemoglobin, $4.9 \mathrm{~g} /$ dL [normal, 13-17 g/dL], hematocrit, 16\% [normal, 35\%-45\%]). A computed-tomography (CT) scan of the abdomen and pelvis with contrast revealed a mass of $6.9 \times 4.7 \times 6.3 \mathrm{~cm}$ in the rectosigmoid colon and a mass of $10.0 \times 12.0 \times 10.7 \mathrm{~cm}$ in the right hepatic lobe consistent with metastatic disease. The patient was taken to the operating room where the rectosigmoid mass was resected completely. The liver mass was deemed unresectable because of its large size, and surgically directed therapy could not be performed. Pathology was consistent with a T3N1 moderately differentiated adenocarcinoma $11 \mathrm{~cm}$ from the anal verge. Further molecular tumor studies revealed wild type $K R A S$ and $N R A S$, as well as a $B R A F$ mutation.

About 4 weeks after the surgery, the patient was seen at our institution for an initial consultation and was noted to have significant anasarca, including 4+ pitting lower extremity edema and scrotal edema. $\mathrm{He}$ complained of dyspnea on exertion, which he attributed to deconditioning. His resting heart rate was found to be 123 beats per minute (normal, 60-100 $\mathrm{bpm}$ ). Jugular venous distention was present. The patient was sent for an urgent echocardiogram, which showed external compression of the right atrium and ventricle by his liver metastasis resulting in tamponade physiology without the presence of any pericardial effusion (Figure 1). A CT of the abdomen and pelvis at that time showed that the liver mass had increased to $17.6 \times 12.1 \times 16.1 \mathrm{~cm}$, exerting pressure on the heart and causing atelectasis of the right middle and lower lung lobes (Figure 2).

\section{Treatment plan}

The patient was evaluated by surgical oncology for resection, but his cardiovascular status placed him at high risk for perioperative complications, so such surgery was not pursued. Radioembolization was considered but not pursued because the process needed to evaluate, plan, and treat was not considered sufficiently timely. We consulted with our radiation oncology colleagues about external beam radiotherapy (EBRT) for rapid palliation. They evaluated the patient and recommended the EBRT, and the patient signed consent for treatment. We performed a CT-based simulation and generated an external beam, linear-accelerator-based treatment plan. The plan consisted of three 15-megavoltage photon fields delivering 3,000 cGy in 10 fractions to the whole liver, with appropriate multileaf collimation blocking to minimize dose to adjacent heart, right lung, and bilateral kidneys (Figure 3).

Before initiation of the EBRT, the patient received systemic chemotherapy with a doseadjusted FOLFOX regimen (5-FU bolus $200 \mathrm{mg} /$ $\mathrm{m}^{2}$, leucovorin $200 \mathrm{mg} / \mathrm{m}^{2}$, oxaliplatin $85 \mathrm{mg} / \mathrm{m}^{2}$, with infusional 5 -FU $2,400 \mathrm{mg} / \mathrm{m}^{2}$ over 46 hours).

Accepted for publication May 23, 2017. Correspondence: Aditya N Halthore, MD; aditya.halthore@gmail.com. Disclosures: The authors report no disclosures or conflicts of interest. JCSO 2018;16(1):e37-e39. (02017 Frontline Medical Communications. doi: https://doi.org/10.12788/jicso.0352 


\section{Case Report}

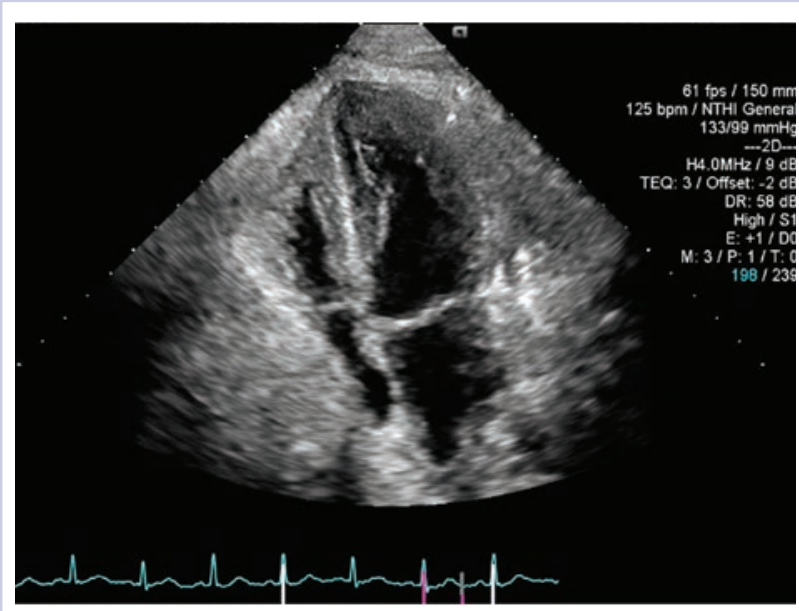

FIGURE 1 Echocardiogram showing extrinsic compression of the heart resulting in tamponade physiology.
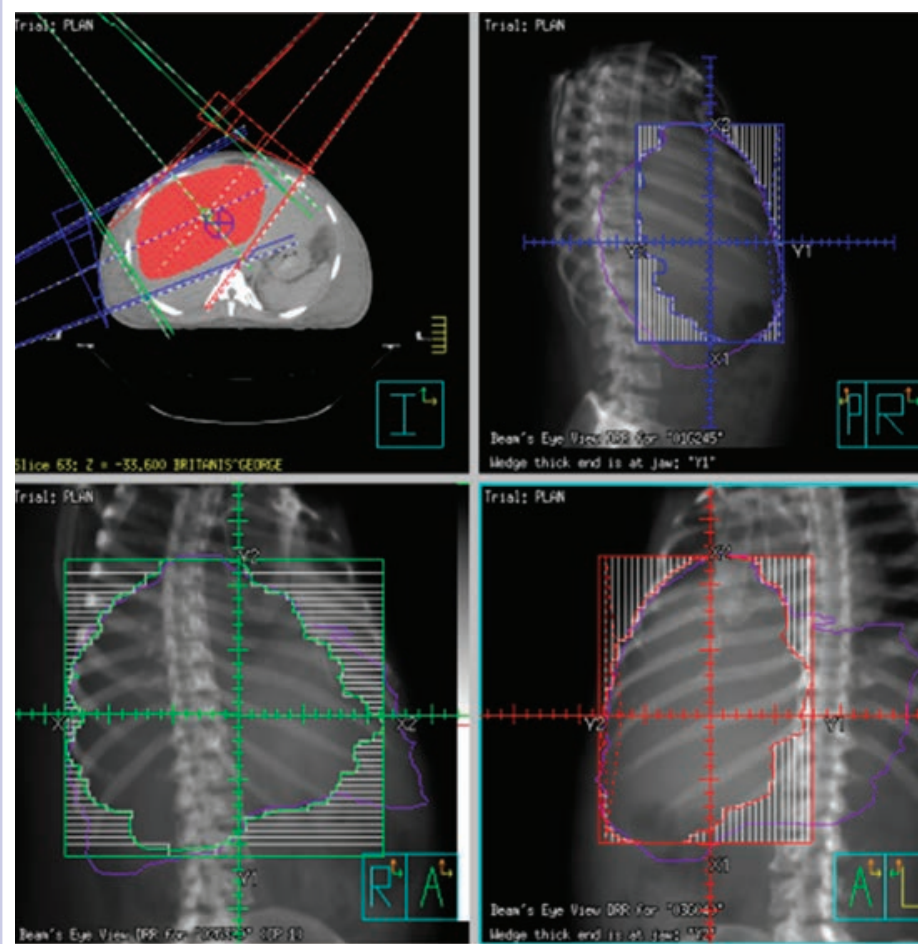

FIGURE 3 Three-field radiation treatment plan, including beam's eye views from one posterior oblique and two anterior oblique fields with blocking to minimize adjacent heart, lung, and kidney dose.

After completing 1 dose of modified FOLFOX, he completed 10 fractions of whole liver radiotherapy with the aforementioned plan. He tolerated the initial treatment well and his subjective symptoms improved. The patient then proceeded to further systemic therapy. After recent data demonstrated improved median progression-free survival and response rates with FOLFOXIRI plus bevaci-

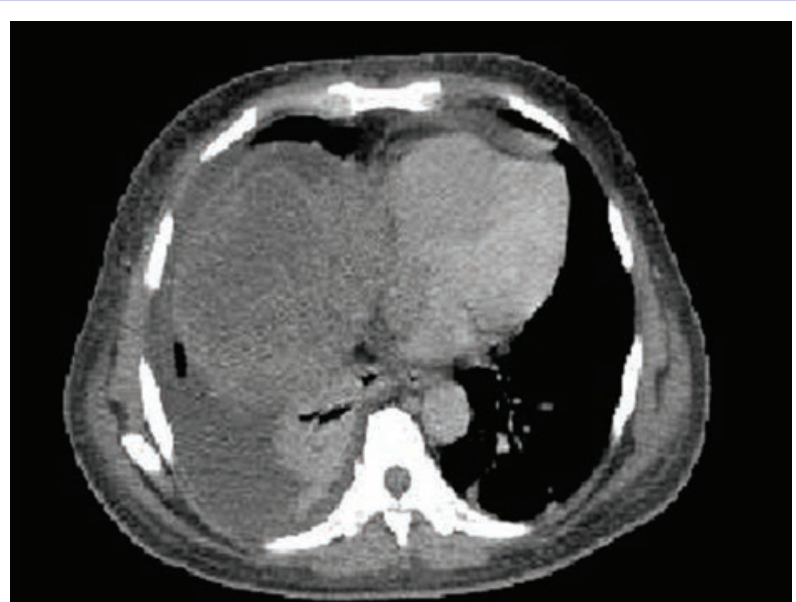

FIGURE 2 Computed-tomography scan demonstrating right middle and lower lobe atelectasis and right ventricular compression by liver mass.

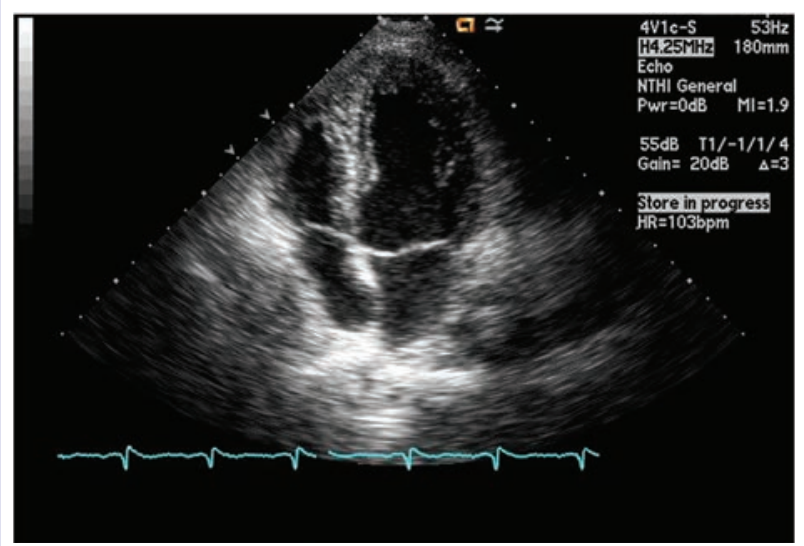

FIGURE 4 Follow-up echocardiogram showing resolution of tamponade.

zumab (infusional 5-FU $3200 \mathrm{mg} / \mathrm{m}^{2}$, leucovorin $200 \mathrm{mg} /$ $\mathrm{m}^{2}$, irinotecan $165 \mathrm{mg} / \mathrm{m}^{2}$, and oxaliplatin $85 \mathrm{mg} / \mathrm{m}^{2}$, bevacizumab $5 \mathrm{mg} / \mathrm{kg}$ ) versus FOLFIRI plus bevacizumab, ${ }^{3}$ we decided to modify his systemic therapy to FOLFOXIRI with bevacizumab to induce a better response.

\section{Treatment response}

After 2 doses of chemotherapy and completion of radiotherapy, the edema and shortness of breath improved. A follow-up echocardiogram performed a month after completion of EBRT, 1 dose of FOLFOX, and 1 dose of FOLFOXIRI showed resolution of the cardiac compression (Figure 4). A CT scan of the abdomen and pelvis obtained after 3 cycles of FOLFOXIRI showed marked decrease in the size of the right lobe hepatic mass from $17.6 \times 12.1 \mathrm{~cm}$ to $12.0 \times 8.0 \mathrm{~cm}$. Given the survival benefit of VEGF inhibition in colon cancer, bevacizumab $(5 \mathrm{mg} /$ $\mathrm{kg}$ ) was added to the FOLFOXIRI regimen with cycle 4. 
Unfortunately, after the $5^{\text {th }}$ cycle, a CT scan of the abdomen showed an increase in size of the hepatic lesions. At this time, FOLFOXIRI and bevacizumab were stopped, and given the tumor's KRAS/NRAS wild type status, systemic therapy was changed to panitumumab $(6 \mathrm{mg} / \mathrm{kg})$. The patient initially tolerated treatment well, but after 9 cycles, the total bilirubin started to increase. $\mathrm{CT}$ abdomen at this point was consistent with progression of disease. The patient was not eligible for a clinical trial targeting $B R A F$ mutation given the elevated bilirubin. Regorafanib ( $80 \mathrm{mg}$ daily for 3 weeks on and 1 week off) was started. After the first cycle, the total bilirubin increased further and the regorafanib was dose reduced to $40 \mathrm{mg}$ daily. Unfortunately, a repeat $\mathrm{CT}$ scan of the abdomen demonstrated progression of disease, and given that he developed a progressive transaminitis and hyperbilirubinemia, hospice care was recommended. The patient died shortly thereafter, about 15 months after his initial diagnosis.

\section{Discussion}

Massive liver metastasis in the setting of disseminated cancer is not an uncommon manifestation of advanced cancer that can have life-threatening consequences. In te present case, a bulky liver metastasis caused extrinsic compression of the right atrium, resulting in obvious clinical and echocardiogram-proven cardiac tamponade physiology. To our knowledge, this is the first reported case of the treatment of a bulky hepatic metastasis causing cardiac tamponade. In this patient's case, both radiotherapy and chemotherapy were given safely in rapid sequence resulting in quick resolution of the patient's

\section{References}

1. American Cancer Society. Cancer Facts \& Figures 2015. https://www. cancer.org/research/cancer-facts-statistics/all-cancer-facts-figures/ cancer-facts-figures-2015.html. Published 2015. Accessed October 10, 2017.

2. Siegel R, Desantis C, Jemal A. Colorectal cancer statistics, 2014. CA Cancer J Clin. 2014;64(2):104-117.

3. Loupakis F, Cremolini C, Masi G, et al. Initial therapy with FOLFOXIRI and bevacizumab for metastatic colorectal cancer. N Engl J Med. 2014;371:1609-1618.

4. Russell AH, Clyde C, Wasserman TH, Turner SS, Rotman M. Accelerated hyperfractionated hepatic irradiation in the management of patients with liver metastases: results of the RTOG dose escalating protocol. Int J Radiat Oncol Biol Phys. 1993;27(1):117-123.

5. Turek-Maischeider M, Kazem I. Palliative irradiation for liver metastases. JAMA. 1975;232(6):625-628.

6. Sherman DM, Weichselbaum R, Order SE, Cloud L, Trey C, Piro AJ Palliation of hepatic metastasis. Cancer. 1978;41(5):2013-2017. symptoms and echocardiogram findings. The presence of a $B R A F$ mutation conferred a poor prognosis and poor response to systemic chemotherapy. Nevertheless, the patient showed good response to a FOLFOXIRI regimen, chosen in this emergent situation given its significantly higher response rates compared with the standard FOLFIRI regimen, which was tolerated well with minimal adverse effects.

Findings from randomized controlled trials examining the role of palliative radiotherapy for metastatic liver disease have suggested that dose escalation above $30 \mathrm{~Gy}$ to the whole liver may lead to unacceptably high rates of radiation-induced liver disease, which typically leads to mortality. ${ }^{4-8}$ Two prospective trials comparing twice daily with daily fractionation have shown no benefit to hyperfractionation, with possibly increased rates of acute toxicity in the setting of hepatocellular carcinoma., ${ }^{9,10}$ There is emerging evidence that partial liver irradiation, in the appropriate setting in the form of boost after whole-liver RT or stereotactic body radiotherapy, may allow for further dose escalation while avoiding clinical hepatitis. ${ }^{11}$ Although there is no clear consensus about optimal RT dose and fractionation, the aforementioned studies show that dose and fractionation schemes ranging between $21 \mathrm{~Gy}$ and $30 \mathrm{~Gy}$ in 1.5 Gy to 3 Gy daily fractions likely provide the best therapeutic ratio for whole-liver irradiation.

In conclusion, this case demonstrates the resolution of cardiac tamponade from a massive liver colorectal metastasis after chemoradiation and illustrates the potential utility of adding radiotherapy to chemotherapy in an urgent scenario where the former might not typically be considered.

7. Prasad B, Lee MS, Hendrickson FR. Irradiation of hepatic metastases. Int J Radiat Oncol Biol Phys. 1977;2:129-132.

8. Borgelt BB, Gelber R, Brady LW, Griffin T, Hendrickson FR. The palliation of hepatic metastases: results of the Radiation Therapy Oncology Group pilot study. Int J Radiat Oncol Biol Phys. 1981;7(5):587-591.

9. Raju PI, Maruyama Y, DeSimone P, MacDonald J. Treatment of liver metastases with a combination of chemotherapy and hyperfractionated external radiation therapy. Am J Clin Oncol. 1987;10(1):41-43.

10. Stillwagon GB, Order SE, Guse C, et al. 194 hepatocellular cancers treated by radiation and chemotherapy combinations: toxicity and response: a Radiation Therapy Oncology Group Study. Int J Radiat Oncol Biol Phys. 1989;17(6):1223-1229.

11. Mohiuddin M, Chen E, Ahmad N. Combined liver radiation and chemotherapy for palliation of hepatic metastases from colorectal cancer. J Clin Oncol. 1996;14(3):722-728. 